\title{
Trends in der Rheumaorthopädie
}

Esin $-s$ ist offensichtlich, daß auch die Orthopädie sich dem Trend zur Subspezialisierung nicht entziehen kann und auch nicht entziehen soll. Die Erkenntnisse, wie sie sich aus der Grundlagenforschung ergeben, können in immer schnellerem Maße in die klinische Anwendung überführt werden und stehen so, dank der uns zur Verfügung stehenden Kommunikationsmöglichkeiten einem weiten Kreis von behandelnden Ärzten rasch zur Verfügung. Es kann nicht ausbleiben, daß sich mit dieser Informationsflut eine Subspezialisierung aufbesondere Gelenkbereiche, sei es die Hand, die Wirbelsäule oder die untere Extremität von selbst notwendig macht, will man aufdiesen einzelnen Gebieten den notwendigen Wissensstand behalten. So zeigt sich auch in dem hier vorgelegten Heft, wie wichtig es ist, daß der chirurgisch Tätige ein hohes Ausmaß an Erfahrung besitzt, um eine optimale Behandlung gewährleisten zu können.

Dennoch bleibt es der Rheumachirurgie vorbehalten, daß sie mehr verlangt als eine gelenkbezogene Behandlung, wie sie von jedem Spezialisten ausgeführt werden könnte. Die zunächst konservative Behandlung dieses Leidens verlangt nach dem spezialisierten Rheumatologen oder einem entsprechend ausgebildeten Allgemeinarzt oder Internisten, der sich sowohl in den Grundlagen, wie auch in den verschiedenen zur Verfügung stehenden Therapiemöglichkeiten auskennt. Diese mögen zunächst auf die medikamentöse Therapie mit verschiedenen nichtsteroidalen Antirheumatika beschränkt sein, die dann in die spezielle medikamentöse Therapie weiterführen, wobei ein Grundverständnis der Pharmakologie für diese Therapie unumgänglich ist. Mit der Radiosynoviorthese steht ein weiteres Therapieverfahren zur Verfügung, das aber sicherlich nicht routinemäßig angewandt werden sollte, sondern es bleibt dem Erfahrenen überlassen, den Zeitpunkt und die Gelenke zu bestimmen, die mit einer solchen Therapie, sei es alleinig oder in Kombination mit einem chirurgischen Eingriff, sinnvoll behandelt werden können.

Schließlich wird der chirurgisch tätige Orthopäde im Rheumatiker nicht nur ein Gelenk allein therapieren können, handelt es sich doch um eine vielfacettige Erkrankung, wie der Name Polyarthritis treffend ausweist. Es wird dem Rheumachirurgen überlassen bleiben, gleich ob er einen Eingriff selbst durchführt oder einem subspezialisierten Fachkollegen weiterleitet, für seinen Patienten einen Behandlungsplan aufzustellen, mit dem er ihm die Möglichkeit gibt, seine Mobilität und damit seinen Lebensraum möglichst lange zu erhalten.

So soll dieses Heft nicht eine Ansammlung von subspezialisierten Artikeln sein, sondern als Teil eines Mosaiks verstanden werden, aus dem sich die Rheumachirurgie zusammensetzt.

J.F. Löhr • B. R.Simmen •

N. Gschwend

Die Herausgeber 